\title{
Treinamento Físico na Síndrome Lipodistrófica: Revisão Sistemática
}

\author{
Physical Training in the Lipodistrophy Syndrome: a Systematic Review
}

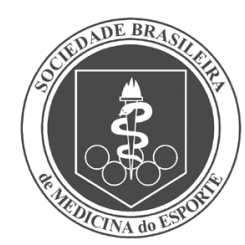

Artigo de ReVISÃo
Gabriela Maria Viega Juchem ${ }^{1}$ Alexandre Ramos Lazzarotto ${ }^{2}$

1. Fisioterapeuta do Programa de Aperfeiçoamento Científico do Centro Universitário Feevale.

2. Professor e Pesquisador do Centro Universitário Feevale; Doutor em Ciências do Movimento Humano pela Universidade Federal do Rio Grande do Sul.

\section{Endereço para correspondência:} Campus II - Sala 201K - Prédio Lilás RS - 239, 2.755

93352-000 - Novo Hamburgo, RS E-mail: gabijuchemnh@hotmail.com

\begin{abstract}
RESUMO
Com a cronicidade da infecção pelo HIV, a partir do uso da terapia antirretroviral combinada (TARV), o indivíduo torna-se predisposto às alterações metabólicas e corporais denominadas síndrome lipodistrófica, que têm como consequência o risco aumentado para doenças cardiovasculares. Tornam-se necessários estudos com viabilidades terapêuticas com o intuito de melhorar a qualidade de vida destes pacientes. Neste contexto, o treinamento físico deve ser abordado como terapêutica para minimizar os efeitos deletérios da TARV. O objetivo desse artigo foi revisar na literatura as evidências científicas sobre o treinamento físico na síndrome lipodistrófica. O método utilizado foi uma revisão sistemática com a localização de artigos publicados entre 2000 e 2008 nas bases de dados PubMed e SciELO. As evidências científicas encontradas nos estudos analisados indicam que a maioria dos estudos, independente do tipo de treinamento, obtiveram melhoria significativa na composição corporal. Dessa forma, tornam-se necessário mais estudos investigando o treinamento físico no perfil lipídico, na resistência a insulina e nas alterações glicêmicas em pessoas vivendo com HIV/Aids.
\end{abstract}

Palavras-chave: treinamento físico, síndrome lipodistrófica, HIV.

\begin{abstract}
The chronicity of the HIV infection from the use of the highly active antiretroviral therapy (HAART), the subject becomes prone to metabolic and physical abnormalities called Lipodystrophy Syndrome, which has as consequences increased risk of cardiovascular diseases. Therefore, there is a need for therapeutical studies in order to improve the quality of life of these patients. In this context, physical training should be approached as a therapy to minimize the TARV deleterious effects. The aim of this article was to review in the literature the evidence about physical training in the Lipodystrophy Syndrome. The used method was a systematic review with papers published between 2000 and 2008, in PubMed and Scielo databases. The scientific evidence found in the assessed studies indicates that most of the studies, regardless of the training type, had significant improvement in body composition. Thus, further studies on lipid status, insulin resistance and glucose alterations by physical training on subjects living with HIV are necessary.
\end{abstract}

Keywords: physical training, lipodystrophy syndrome, HIV.

\section{INTRODUÇÃO}

A Aids é estágio final da infecção pelo HIV, o qual é transmitido pelas vias sexual, parenteral e vertical. Ela caracteriza-se pela imunossupressão profunda, especialmente da imunidade celular que, na maioria dos casos, evolui para infecções oportunistas, neoplasias secundárias e manifestações neurológicas ${ }^{(1-3)}$. No Brasil, o critério utilizado para definir casos de Aids em indivíduos a partir de 13 anos é a contagem de T-CD4+ abaixo de 350 cópias/ml e ou as infecções oportunistas ${ }^{(4)}$.

De acordo com o boletim epidemiológico da Unaids 2008, o número de casos com Aids no mundo é de 33,2 milhões com índice de mortalidade de 2,1 milhões ${ }^{(5)}$. No Brasil foram notificados de 1980 a junho de 2008, 506 mil casos de Aids ${ }^{(6)}$, com a taxa de prevalência de 0,61\% na população de 15 a 49 anos, sendo que, 0,42\% são do sexo feminino e $0,82 \%$ do masculino ${ }^{(7)}$.

Desde 1996, a terapia antirretroviral combinada (TARV), com a inclusão dos inibidores da protease (IP), proporcionou a supressão sustentada da carga viral e a restauração da resposta imunológica, proporcionado, desta forma, menor morbidade e mortalidade. Atualmente, a Aids é doença crônica, ou seja, controlada por farmacoterapia ${ }^{(2,4)}$. No
Brasil, a média de sobrevida que era de 58 meses, entre 1995 e 1996, aumentou para 108 meses, entre 1998 e 1999, havendo a diminuição em $50 \%$ no número de óbitos. Aproximadamente $94,8 \%$ dos pacientes com Aids estão recebendo a TARV (95,3\% são mulheres e 94,5\% são homens) $)^{(7)}$. Até o final de 2008 estima-se que 180.000 brasileiros a receberam no âmbito do acesso universal ao tratamento, disponibilizada pelo Sistema Único de Saúde (SUS)(4).

Mesmo com o aumento da sobrevida dos indivíduos com Aids, a comunidade científica depara-se com a instalação de outras comorbidades, que podem apresentar impacto na qualidade de vida dos pacientes, destacando-se dentre elas, a síndrome lipodistrófica, caracterizada pela redistribuição de gordura e ou alterações metabólicas, que têm como consequência o risco aumentado para doenças cardiovasculares ${ }^{(2,4,8)}$. Devido a esse aumento, é recomendado que os pacientes adotem estratégias saudáveis, como, por exemplo, seguir um controle nutricional, evitar o tabagismo e praticar exercício físico ${ }^{(2,4)}$. É importante também o acompanhamento de equipe multidisciplinar, composta por infectologista, endocrinologista, cardiologista, nutricionista, profissional de educação física e fisioterapeuta ${ }^{(4,9,10)}$. 
Considerando o treinamento físico, ou seja, o exercício crônico, existe carência de estudos quanto a sua viabilidade terapêutica nas alterações corporais e metabólicas. Sendo assim, o objetivo desse artigo consiste em revisar, na literatura, as evidências científicas sobre o treinamento físico na síndrome lipodistrófica.

\section{MÉTODO}

O método de abordagem dos treinamentos constituiu-se na revisão sistemática, composta por artigos publicados entre 2000 e 2008, nas bases de dados Pubmed e SciELO. Os unitermos de busca foram exercício físico e HIV, exercício físico e síndrome lipodistrófica, exercise and HIV e exercise and lipodystrophy syndrome, excluindo-se trabalhos de revisão. Os estudos foram classificados de acordo com os níveis de evidência do Center for Evidence Based Medicine de Oxford (WWW.cebm. net): $<1>$ (ensaio clínico randomizado ou revisão sistemática de ensaios clínicos randomizados com desfechos clínicos), <ll> (ensaio clínico randomizado ou revisão sistemática de ensaio clínico de menor qualidade, estudos observacionais ou revisão sistemáticas destes estudos) <III> (ensaio clínico randomizado com desfechos substitutos não-validados) e estudo caso-controle, <IV> (estudo com desfecho clínico, porém, com maior potencial de viés), $\langle\mathrm{V}\rangle$ (fórum representativo ou opinião de especialista sem evidências dos níveis supracitados).

\section{SÍNDROME LIPODISTRÓFICA}

A partir de 1996, com a introdução do IP, a TARV tornou-se rotina no tratamento de pacientes com Aids, diminuindo a replicação do HIV e consequentemente, a presença do RNA do HIV no plasma para níveis indetectáveis ${ }^{(4,8)}$. Em indivíduos HIV assintomáticos, com a contagem de linfócitos T-CD4+ acima de 350/ml não é recomendado iniciar o tratamento, considerando que os benefícios não estão suficientemente claros para contrabalançar com os potenciais riscos da TARV. Indivíduos assintomáticos com contagem de linfócitos T-CD4+ entre 200 e 350/ $\mathrm{ml}$, o tratamento deve ser recomendado. Quanto mais próxima de 200 células/ml estiver a contagem de T-CD4+, maior é o risco de progressão para Aids, especialmente se associada à carga viral plasmática elevada (maior que 100.000 cópias/ml). A decisão de iniciar ou não dependerá da tendência de diminuição da contagem desses linfócitos e/ou elevação da carga viral, da motivação do paciente para iniciar o tratamento, da sua capacidade de adesão e a presença de comorbidades. Em síntese, deve-se iniciar o tratamento com TARV em pacientes assintomáticos com linfócitos T-CD4+ menor que 200 células/ml e sintomáticos com presença de sintomas ou manifestações clínicas associadas à imunodeficiência relacionada ao HIV, mesmo quando não definidoras de Aids, independentemente dos parâmetros imunológicos ${ }^{(4)}$.

Infelizmente, foram observadas alterações corporais e metabólicas em pacientes expostos ao uso prolongado da TARV (a partir de seis meses). O conjunto dessas alterações foi posteriormente denominado de síndrome lipodistrófica, composta pelos seguintes fatores: lipodistrofia, dislipidemia, resistência à insulina e hiperglicemia ${ }^{(1,2,4,8)}$.

A lipodistrofia é a redistribuição da gordura corporal e pode ser classificada clinicamente em três categorias: a lipohipertrofia, que é o acúmulo de gordura na região abdominal, peitoral e na região dorsocervical. O acúmulo de tecido adiposo abdominal é a apresentação mais frequente, levando ao aumento da circunferência abdominal (protease belly) com protusão dos órgãos, causando por vezes desconforto abdominal e/ou flatulência. A lipoatrofia é a diminuição do tecido adiposo subcutâneo, principalmente na face, membros inferiores e glúteos. A lipoatrofia nesses membros causa a diminuição dos seus diâmetros e a proeminência dos vasos (venomegalias). A forma mista é caracterizada pela associação de componentes das duas formas anteriormente descritas. Aparentemente, os homens têm maior tendência a desenvolver lipoatrofia, enquanto as mulheres, lipohipertrofia. As alterações da composição corporal podem ocorrer mesmo em pacientes que iniciaram a terapia antirretroviral precocemente ${ }^{(11-14)}$.

Carr et al. ${ }^{(15)}$ estabeleceram uma teoria que explica a lipodistrofia, fundamentada na homologia entre o sítio catalítico da protease do HIV e as duas proteínas envolvidas no metabolismo lipídico: a CRABP-1 (cytoplasmatic retinoic-acid binding protein type 1 ou proteína ligadora do ácido retinoico citoplasmático tipo 1) e a LRP (low density lipoproteinreceptor related protein ou proteína relacionada ao receptor de LDL). Os inibidores de protease inibem a CRBP-1 e se unem à LRP, impedindo etapas importantes do metabolismo lipídico humano. Como o do sítio da CRBP-1 está ocupado pelos inibidores de protease, não existe a sua ligação ao ácido retinoico, reduzindo a diferenciação de adipócitos e elevando a apoptose de adipócitos periféricos. O resultado é a hiperlipidemia por redução do armazenamento periférico e aumento da liberação de lipídios na corrente sanguínea.

Os métodos utilizados para o diagnóstico da lipodistrofia incluem autoavaliação do paciente, avaliação médica, medidas antropométricas, como, por exemplo, dobras cutâneas, relação cintura/quadril, métodos de imagem como ultrassonografia da região subcutânea, Dexa (dual energy $X$-ray absorptiometry-absorciometria de raios- $X$ de dupla energia), tomografia computadorizada e ressonância magnética nuclear do abdome. A medida da espessura da dobra cutânea é potencialmente útil para monitorar o acúmulo de gordura subcutânea em pacientes com lipoatrofia. A medida da relação cintura/quadril poderia, teoricamente, correlacionar as manifestações da lipoatrofia e da lipohipertrofia; entretanto, a relação cintura/quadril é pouco confiável para o diagnóstico de lipodistrofia em relação a outras medidas antropométricas. A medida da circunferência do quadril per se, parece ter maior sensibilidade e especificidade do que relação cintura/quadril. O Dexa tem sido bastante utilizado no diagnóstico da lipodistrofia $(12,13,16)$

As alterações metabólicas observadas com o uso prolongado da TARV são a dislipidemia, resistência à insulina e hiperglicemia associadas principalmente aos esquemas terapêuticos contendo IP. As alterações lipídicas encontradas nos pacientes infectados pelo HIV são o aumento dos níveis séricos de triglicérides (TG) e/ou de colesterol total (CT), devido às lipoproteínas de baixa densidade (LDL), havendo tendência à diminuição dos níveis de lipoproteínas de alta densidade $(\mathrm{HDL})^{(17-19)}$. Iniciado o tratamento, estima-se que entre $33 \%$ a $82 \%$ dos pacientes desenvolvam hipercolesterolemia, e 43\% a $66 \%$ hipertrigliceridemia(4). De acordo com a classificação do NCEP (National Cholesterol Education Program) ATP III 2002(20) OS valores de referência para CT é $<200 \mathrm{mg} / \mathrm{dL}$, LDL $<100 \mathrm{mg} / \mathrm{dL}, \mathrm{HDL} \geq$ a $60 \mathrm{mg} / \mathrm{dL}$ e TG $<150 \mathrm{mg} / \mathrm{dL}$.

Carr et al. ${ }^{(15)}$ afirmam que a diabetes tipo II é menos comum que a hiperlipidemia e a resistência à insulina em portadores de HIV. O diagnóstico e sintomas de diabetes tipo II em portadores de HIV não se diferenciam em relação à população geral. As alterações metabólicas da glicose são muitas vezes acompanhadas de alterações do metabolismo lipídico e podem levar ao maior risco de doenças cardiovasculares ${ }^{(4)}$.

Atualmente não existe tratamento padrão para nenhum componente da síndrome lipodistrófica e a decisão do tratamento vai depender de algumas variáveis como: presença de sintomas, quadro clínico, tipo de antirretrovirais utilizados, tempo de uso da medicação e presença de um ou mais fatores de risco cardiovascular ${ }^{(4)}$.

A partir dos pressupostos teóricos expostos anteriormente, inferese que é necessário controlar esses fatores de risco à doença cardiovascular (lipodistrofia, dislipidemia, resistência à insulina e hiperglicemia) considerando estratégias viáveis dentro de um contexto de saúde pública, como, por exemplo, modificações no estilo de vida: acompanhamento nutricional e treinamento físico ${ }^{(4,14,17,21)}$. 


\section{TREINAMENTO FÍSICO NA SÍNDROME LIPODISTRÓFICA}

Os estudos apresentados abaixo estão organizados em treinamento aeróbio, treinamento de força e treinamento concorrente (associação dos componentes aeróbios e de força na mesma sessão de treinamento)(22,23), nos fatores que compõem a síndrome lipodistrófica. Os treinamentos com séries múltiplas são compostos de três repetições de cada exercício na mesma sessão.

\section{Treinamentos aeróbio e de força}

Considerando os treinamentos aeróbios e de força, foram localizados quatro estudos: Terry et al. ${ }^{(24)}$, Smith et al. ${ }^{(25)}$, Yarasheski et al. (26) e Lindegaard et al. (27).

Terry et al. ${ }^{(24)}<1>$ em estudo randomizado com 30 pacientes HIV positivos com uso de TARV e dislipidemia, verificaram o $\mathrm{VO}_{2 \text { pico, }}$ a composição corporal, a contagem de T-CD4+, a carga viral e o perfil lipídico em um programa de exercícios aeróbios. Os participantes foram alocados aleatoriamente em dois grupos, nos quais 15 realizavam o treinamento aeróbio e controle nutricional, e 15 somente controle nutricional. O programa transcorreu durante 12 semanas (36 sessões de 60 minutos, três vezes por semana). $\mathrm{OVO}_{2 \text { pico }}$ aumentou significantemente no grupo que realizou o treinamento aeróbio e controle nutricional e a composição corporal melhorou significativamente nos dois grupos, porém, as variáveis imunológicas e o perfil lipídico não obtiveram diferenças significativas.

Smith et al. ${ }^{(25)}<1>$ realizaram estudo randomizado com 60 pacientes HIV positivos (52 homens e oito mulheres) alocados em dois grupos, sendo que 30 eram do grupo experimental e 30 do grupo controle; entretanto, somente 19 participantes do grupo experimental finalizaram o treinamento. Foram avaliadas a fadiga, dispneia, volume expiratório forçado, massa corporal total e composição corporal em programa supervisionado de exercícios aeróbios, sendo que o grupo controle realizava suas atividades habituais. $\mathrm{O}$ treinamento transcorreu durante 12 semanas (três sessões semanais de, no mínimo, 30 minutos de exercícios). Somente 14 pacientes estavam em uso de TARV. Foi identificada a diminuição significativa na fadiga, massa corporal total, IMC (índice de massa corpórea), circunferência abdominal e na gordura subcutânea, ou seja, uma melhoria significativa na composição corporal no grupo experimental. $\mathrm{OVO}_{2 \text { máx }}$ dispneia, T-CD4+ e carga viral não apresentaram diferença significativa em ambos os grupos.

Yarasheski et al. ${ }^{(26)}$, <IV > em um estudo experimental não-controlado, avaliaram o treinamento de força com séries múltiplas em 18 homens HIV positivos em uso de TARV, durante 16 semanas (quatro sessões semanais com duração de uma hora a uma hora e meia). Houve aumento significativo na massa muscular e na força e diminuição significativa nos triglicerídeos, porém, o mesmo não ocorreu nas variáveis CT, HDL e LDL.

Lindegaard et al. ${ }^{(27)}<1>$ avaliaram a resistência à insulina, a composição corporal, a alteração no perfil lipídico e os marcadores inflamatórios, em um estudo randomizado, com 20 homens sedentários HIV positivos com lipodistrofia em uso de TARV. Desses, 10 realizaram treinamento aeróbio e 10 de força (com séries múltiplas), durante 16 semanas (três sessões semanais durante 35 minutos de aeróbio e 45-60 minutos de força). Ambos os treinamentos melhoraram a resistência à insulina e diminuíram os marcadores inflamatórios no plasma (proteína C-reativa, TNF-a, IL-6 e IL-18). Os participantes do treinamento de força obtiveram aumento significativo da massa magra e diminuição da gordura corporal em comparação aos participantes do aeróbio. Houve a diminuição do CT, LDL e aumento do HDL no grupo do treinamento aeróbio, enquanto que no grupo do treinamento de força ocorreu a diminuição dos triglicerídeos e aumento do HDL.

\section{Treinamento concorrente}

Os trabalhos que associaram o treinamento aeróbio ao de força na mesma sessão totalizaram sete estudos: Dolan et al. ${ }^{(28)}$, Driscoll et al. ${ }^{(29)}$,
Driscoll et al. ${ }^{(30)}$, Grinspoon et al. ${ }^{(31)}$, Engelson et al. ${ }^{(32)}$, Jones et al. ${ }^{(33)} \mathrm{e}$ Robinson et al. ${ }^{(34)}$.

Dolan et al. ${ }^{(28)}<1>$ realizaram um estudo randomizado com 40 mulheres portadoras de HIV em uso de TARV, com aumento da relação cintura/ quadril e alteração na redistribuição da gordura. Avaliaram um programa de treinamento concorrente com séries múltiplas nas suas respectivas casas durante 16 semanas (três sessões semanais com duração de 120 minutos), nas quais as participantes do grupo experimental receberam o equipamento necessário. As variáveis avaliadas foram $\mathrm{VO}_{2 \text { máx }}$ força, composição corporal, perfil lipídico, glicose, pressão arterial, T-CD4+ e carga viral. As participantes apresentaram aumento significativo no $\mathrm{VO}_{2 \text { máx }}$ e na força e não apresentaram melhoria significativa na composição corporal.

Driscoll et al. ${ }^{(29)},<1>$ em um estudo randomizado com 25 indivíduos (20 homens e cinco mulheres) em uso de TARV, avaliaram o treinamento concorrente com séries múltiplas associado à metformina durante 12 semanas (três sessões semanais com duração de 60 minutos) na sua composição corporal. Os participantes que utilizaram a metformina associada ao treinamento $(n=11)$ obtiveram diminuição da gordura corporal em relação ao grupo que utilizou somente a metformina.

Driscoll et al. ${ }^{(30)},<1>$ em um segundo estudo com os mesmos indivíduos, treinamento e duração do programa avaliaram a condição cardiovascular, composição corporal, força, perfil lipídico, T-CD4+ e carga viral. O grupo que utilizou a metformina associada ao treinamento ( $\mathrm{n}$ = 11) melhorou significativamente a condição cardiovascular e a força, comparado ao grupo que utilizou somente metformina, porém não houve diferença significativa em perfil lipídico, massa corporal total, índice de massa corpórea, T-CD4+ e carga viral.

Grinspoon et al. ${ }^{(31)}<1>$ realizaram um estudo randomizado com 54 homens eugonadais em uso de TARV que apresentavam wasting syndrome. Investigaram o treinamento concorrente com séries múltiplas associado à testosterona (grupo experimental) comparado ao treinamento e placebo (grupo controle) durante 12 semanas (três sessões semanais, não foi informado o tempo das sessões). Foi observado aumento significativo na massa muscular e diminuição significativa na carga viral no grupo experimental. Em ambos os grupos, o T-CD4+ não apresentou aumento significativo.

Engelson et al. ${ }^{(32)}$, <IV > em um estudo experimental não-controlado, avaliaram o treinamento concorrente com séries múltiplas associado à dietoterapia na composição corporal, condição cardiovascular, força, aspectos metabólicos e qualidade de vida de 18 mulheres obesas em uso de TARV. As participantes foram acompanhadas durante 19 meses (três sessões semanais com duração de 90 minutos) e apresentaram melhoria da composição corporal e aumento significativo no $\mathrm{VO}_{2 \text { relativo }}$ e força. Não houve diferença significativa em T-CD4+ e carga viral.

Jones et al. ${ }^{(33)}<$ IV $>$ realizaram um estudo experimental não-controlado com cinco homens e uma mulher com lipodistrofia durante 10 semanas (três sessões com duração de 90 minutos) de treinamento concorrente com séries múltiplas. Obtiveram melhorias significativas no perfil lipídico (diminuição do CT e triglicerídeos) e na composição corporal (aumento da massa muscular e diminuição da gordura corporal) e aumento significativo na força e capacidade cardiorrespiratória.

Robinson et al. ${ }^{(34)}$, <IV > em um estudo experimental não controlado, avaliaram a gordura corporal, dislipidemia e a resistência à insulina em um programa de exercícios aeróbios (20 minutos) e de força, com séries simples (oito-10 repetições), durante 16 semanas. Foram realizadas três sessões por semana de treinamento aeróbio e duas de força. Os cinco participantes tiveram diminuição significativa da gordura corporal e diminuição dos triglicerídeos e da resistência a insulina; no entanto, não foram significativas.

O quadro 1 apresenta a síntese dos resultados descritos nos treinamentos aeróbio, força e concorrente. 
Quadro 1. Síntese dos resultados dos treinamentos aeróbio, de força e concorrente.

\begin{tabular}{|c|c|c|c|c|c|c|}
\hline \multirow{2}{*}{$\mathbf{N}^{\circ}$} & \multirow{2}{*}{ Treinamento } & \multirow{2}{*}{ Autor e ano } & \multicolumn{4}{|c|}{ Parâmetros da síndrome lipodistrófica } \\
\hline & & & Lipodistrofia & Dislipidemia & Resistência à insulina & Alterações glicêmicas \\
\hline 1 & Aeróbio & Terry et al. (2006) & $\begin{array}{l}\text { Melhoria da composição corporal } \\
2 \text { grupos }\end{array}$ & = perfil lipídico & NA & NA \\
\hline 2 & Aeróbio & Smith et al. (2001) & Melhoria da composição corporal & NA & NA & NA \\
\hline 3 & Força & Yarasheski et al. (2001) & $\uparrow$ massa muscular & $\downarrow$ triglicerídeos & NA & NA \\
\hline \multirow{2}{*}{4} & Aeróbio & \multirow{2}{*}{ Lindegaard et al. (2008) } & Não obteve melhoria significativa & $\begin{array}{c}\downarrow C T \text { e } L D L \\
\uparrow \mathrm{HDL} \\
\end{array}$ & Melhoria significativa & NA \\
\hline & Força & & $\begin{array}{c}\uparrow \text { massa magra } \\
\downarrow \text { gordura corporal }\end{array}$ & $\begin{array}{c}\downarrow \text { triglicerídeos } \\
\uparrow \mathrm{HDL} \\
\end{array}$ & Melhoria significativa & NA \\
\hline 5 & Concorrente & Dolan et al. (2006) & Não obteve melhoria significativa & $=$ perfil lipídico & NA & $=$ glicose \\
\hline 6 & Concorrente & Driscoll et al. (2004) & $\downarrow$ gordura corporal & NA & NA & NA \\
\hline 7 & Concorrente & Driscoll et al. (2004) & Não obteve melhoria significativa & = perfil lipídico & NA & NA \\
\hline 8 & Concorrente & Grinspoon et al. (2000) & $\uparrow$ massa muscular & NA & NA & NA \\
\hline 9 & Concorrente & Engelson et al. (2006) & Melhoria da composição corporal & NA & NA & NA \\
\hline 10 & Concorrente & Jones et al. (2001) & $\begin{array}{l}\uparrow \text { massa muscular } \\
\downarrow \text { gordura corporal }\end{array}$ & $\downarrow C T$ e triglicerídeos & NA & NA \\
\hline 11 & Concorrente & Robinson et al. (2007) & $\downarrow$ gordura corporal & $=$ perfil lipídico & $=$ resistência à insulina & \\
\hline
\end{tabular}

NA - não avaliado; = - não houve diferença significativa.

Considerando as alterações metabólicas e corporais da síndrome lipodistrófica associadas aos resultados dos estudos de treinamento físico, pode-se afirmar que:

a) Os dois estudos de treinamento aeróbio obtiveram melhoria significativa na composição corporal.

b) $\mathrm{O}$ estudo de treinamento de força melhorou significativamente a composição corporal e a diminuição dos triglicerídeos.

c) No estudo que comparou o treinamento aeróbio com o de força houve melhoria significativa da composição corporal apenas no treinamento de força e, em ambos, observou-se melhoria significativa no perfil lipídico e na resistência à insulina.

d) Em quatro, dos seis estudos de treinamento concorrente com séries múltiplas, observou-se melhoria significativa na composição corporal e em apenas um verificou-se melhoria significativa no perfil lipídico.

\section{REFERÊNCIAS}

1. Hoffman C, Rockstroh JK, Kamps BS. HIV Medicine-2007. Disponível em http://www.hivmedice.com. Acesso em 05 de setembro de 2009 .

2. Valente AMM, Reis AF, Machado DM, Succi RCM, Chacra AR. Alterações metabólicas da síndrome lipodistrófica do HIV. Arq Bras Endocrinol Metab 2005:49:871-81.

3. Canini SR, Reis RB, Pereira LA, et al. Qualidade de vida de indivíduos com HIV/Aids: Uma revisão de literatura. Revista Latino-Americana de Enfermagem 2004;12:940-5.

4. Brasil. Ministério da Saúde. Recomendaçöes para terapia antirretroviral em adultos e adolescentes infectados pelo HIV-2007/2008. Disponível em <http://www.aids.gov.br>. Acesso em 18 de agosto de 2009.

5. UNAIDS. AIDS epidemic update 2008. Disponível em <http//www.unaids.org $>$. Acesso em 17 de setembro de 2009

6. Brasil. Ministério da Saúde. Boletim Epidemiológico - AIDS e DST-2008. Brasíllia, DF. Disponivel em <http:// www aids gov br>. Acesso em 17 de setembro de 2009

7. Simão M. Ministério da Saúde. Desafios Contemporâneos no Enfrentamento do HIV e da Aids no Brasil. Programa Nacional de DST/Aids, 2009.

8. Robinson FP. HIV Lipodystrophy Syndrome: a primer. J Assoc Nurses Aids Care 2004;15:15-29.

9. Guimaraes MMM, Greco DB, Júnior ARO, Penido AG, Machado LC. Distribuição da gordura corporal e perfil lipídico e glicêmico de pacientes infectados pelo HIV. Arq Bras Endocrinol Metab 2007;51:42-51.

10. Cade WT, Peralta L, Keyser RE. Aerobic exercise dysfunction in human immunodeficiency virus: a potential link to physical disability. Phys Ther 2004;84:655-65.

11. Chen D, Misra A, Gard A. Lipodystrophy in Human Immunodeficiency Virus- Infected Patients. J Clin Endocrinol Metab 2002;87:4845-56.

12. Herrans P, Lucas R, Pérez-Espana L, Mayor M. Lipodystrophy Syndromes. Dermatol Clin 2008;26:569-78.

13. Bedimo RJ. Body-Fat Abnormalities in Patients With HIV: Progress and Challenges. J Int Assoc Physicians AIDS Care (Chic III) 2008;7:292-305.

14. Cofracesco J, Freedland E, Mccomsey G. Treatment Options for HIV Associated Central Fat Accumulation. AIDS Patient Care STDS 2009;23:5-18

15. Carr A, Samaras K, Chisholm DJ, et al. Pathogenesis of HIV-1-protease inhibitor-associated peripheral lipodystrofy, hyperlipidaemia, and insulin resistance. Lancet 1998;352:1881-3.

16. Mallewa JE, Wilkins E, Vilari J, Mallewa M, Doran D, et al. HIV-associated lipodystrophy: a review of underlying mechanisms and therapeutic options. J Antimicrob Chemother 2008;62:648-60.

17. Samaras $\mathrm{K}$, et al. Metabolic consequences and therapeutic options in highly active antiretroviral therapy in human immunodeficiency virus-1 infection. J Antimicrob Chemother 2008;61:238-45.

18. Khunnawat C, Mukerji S, Havlichek D, Touma R, Abela GS. Cardiovascular Manifestations in Human Immunodeficiency Virus-Infected Patients. Am J Cardiol 2008;102:635-42. e) No estudo de treinamento concorrente com séries simples houve melhoria significativa na composição corporal.

Em síntese, na maioria dos estudos, independente do tipo de treinamento, ocorre a melhoria da composição corporal de pessoas vivendo com HIV/Aids em uso de TARV.

\section{CONCLUSÃO}

Tendo em vista que a maioria dos resultados dos estudos analisados demonstram melhoria significativa somente na composição corporal, tornam-se necessários mais estudos para investigar o treinamento físico no perfil lipídico, na resistência à insulina e nas alterações glicêmicas em pessoas vivendo com HIV/Aids.

Todos os autores declararam não haver qualquer potencial conflito de interesses referente a este artigo.

19. Calza L, Manfredi R, Chiodo F. Dyslipidaemia associated with antiretroviral therapy in HIV-infected patiens. J Antimicrob Chemother 2004;53:10-4.

20. NCEP. National Cholesterol Education Program ATP III 2002. Disponível em < http://www.ncep.noaa.gov/> Acesso em 19 de outubro de 2009

21. Ciccolo JT, Jowers EM, Bartholomew JB. The Benefits of Exercise Training for Quality of Life in HIV/AIDS in the Post-HAART Era. Sports Medicine 2004;38:487-99.

22. Bell GJ, Syrotuik D, Martin TP, Burnham R, Quinney HA. Effect of concurrent strength and endurance training on skeletal muscle properties and hormone concentrations in humans. Eur J Appl Physiol 2000;8:418-27.

24. Terry L, Sprinz E, Stein R, Medeiros NB, Oliveira J, et al. Exercise training in HIV-1-infected individuals with dyslipidemia and lipodystrophy. Medicine and Science in Sports Exercise 2006;38:411-7.

25. Smith BA, Neidig JL, Nickel JT, Mitchell GL, Para MF, Fass RJ. Aerobic exercise: effects on parameters related to fatigue, dyspnea, weight and body composition in HIV-infected adults. AIDS 2001;15:693-701.

26. Yarasheski KE, Pablo T, Barbara S, Sherry C, Donna M, Kyongtae B, et al. Resistance exercise training reduces hypertriglyceridemia in HIV-infected men treated with antiviral therapy. Journal of Applied Physiology 2001;90:133-8.

27. Lindegaard B, Hansen T, Hvid T, Van Hall G, et al. The effect of strength and endurance training on insulin sensitivity and fat distribution in human immunodeficiency virus-infected patients with lipodystrophy. Journal of Clinical Endocrinology and Metabolism 2008:93:3860-9.

28. Dolan SE, Frontera W, Librizzi J, Ljungquist K, Juan S, Dorman R, et al. Effects of a supervised home-based aerobic and progressive resistance training regimen in women infected with human immunodeficiency virus: a randomized trial. Arch Intern Med 2006;166:1225-31.

29. Driscoll SD, Meininger GE, Ljungquist K, Hadigan C, Torriani M, Klibanski A, et al. Differential effects of metformin and exercise on muscle adiposity and metabolic indices in human immunodeficiency virus - infected patiens. J Clin Endocrinol Metab 2004:89:2171-8.

30. Driscoll SD, Meininger GE, Ljungquist K, Hadigan C, Torriani M, Klibanski A, et al. Effects of exercise training and metformina on body composition and cardiovascular indices in HIV - infected patiens. AIDS 2004:18:465-73.

31. Grinspoon S, Corcoran C, Parlman K, Costello M, Rosenthal D, Anderson E, et al. Effects of testosterone and progressive resistance training in eugonadal men with AIDS wasting. A randomized, controlled trial. Ann intern Med 2000;133:348-55.

32. Engelson ES, Agin D, Kenya S, et al. Body composition and metabolic effects of a diet and exercise weight loss regimen on obese, HIV-infected women. Metabolism Clinical and Experimental 2006;55:1327-36.

33. Jones SP, Doran DA, Leatt PB, Maher B, et al. Short term exercise training improves body composition and hyperlipidemia in HIV-positive individuals with lypodistrophy. AIDS 2001;15:2049-51.

34. Robinson FP, Quinn LT, Rimmer JH. Effects of high-intensity endurance and resistance exercise on HIV metabolic abnormalities: a pilot study. Biol Res Nurs 2007:8:177-85. 